\title{
COMPOSITION DU COMITÉ DES TRAVAUX
DE L'ACADÉMIE DES TECHNOLOGIES
}

\section{Président :}

\section{Yves FARGE}

Membre de l'Académie des technologies - Président du Comité des travaux de I'Académie des technologies - Ancien Directeur de la R \& D du Groupe Pechiney

- Consultant

\section{Membres :}

\section{Michel BOUTHIER}

Membre de l'Académie des technologies - Ingénieur en chef de l'Armement Secrétaire général du Conseil scientifique de la Défense

\section{Philippe COIFFET}

Membre de l'Académie des technologies - Ancien Directeur de recherche au CNRS

\section{Louis DUBERTRET}

Membre de l'Académie des technologies - Président de la Fondation René Touraine pour le progrès thérapeutique en Dermatologie - Directeur de l'Institut de recherche sur la peau

\section{Dominique FERRIOT}

Membre de l'Académie des technologies - Professeure des Universités au Cnam

\section{Erol GELENBE}

Membre de l'Académie des technologies - Professor in the Dennis Gabor Chair - Imperial College

\section{François LEFAUDEUX}

Membre de l'Académie des technologies - Ingénieur général de l'Armement

\section{Jean-Claude MILLET}

Membre de l'Académie des technologies - Fondateur de la société IMAJE Président du Conseil de surveillance de OSMOOZE SA et MILLET Innovation SA

\section{Alain MONGON}

Membre de l'Académie des technologies - Gérant de la société Amisa 


\section{Pierre MONSAN}

Membre de l'Académie des technologies - Professeur à l'Institut national des sciences appliquées de Toulouse et à l'École nationale supérieure des mines de Paris - Membre de l'Institut universitaire de France - Président du CCRRDT MidiPyrénées

\section{Alan PAVÉ}

Membre de l'Académie des technologies - Professeur des Universités - Directeur de recherche au CNRS - Directeur CNRS - Guyane

\section{Gérard SABAH}

Membre de l'Académie des technologies - Directeur de recherche au CNRS

\section{Jean-Paul TEYSSANDIER}

Membre de l'Académie des technologies - Ancien Directeur de VINCI Concessions 